\title{
Ochratoxin A-Induced Renal Cortex Fibrosis and Epithelial-to- Mesenchymal Transition: Molecular Mechanisms of Ochratoxin A-Injury and Potential Effects of Red Wine
}

\author{
Nicoletta Gagliano, ${ }^{1}$ Carlo Torri ${ }^{1}$ Elena Donetti ${ }^{1}{ }^{1}$ Fabio Grizzi ${ }^{2}$ Francesco Costa, ${ }^{1}$ \\ Alberto AE Bertelli, ${ }^{1}$ Massimiliano Migliori, ${ }^{3}$ Cristina Filippi, ${ }^{3}$ Marzia Bedoni, ${ }^{1}$ \\ Vincenzo Panichi, ${ }^{3}$ Luca Giovannini, ${ }^{3}$ AND Magda Gioia ${ }^{1}$
}

\begin{abstract}
We characterized the effect of chronic ochratoxin A (OTA) on rat kidney cortex, analyzing collagen content and collagen turnover and the major markers of epithelial-to-mesenchymal transition (EMT), such as $\alpha$-smooth muscle actin ( $\alpha$ SMA), cadherins, and MMP-9. Because OTA nephrotoxicity is mediated by free radicals, we also investigated whether antioxidants in red wine provided protection for the kidney and attenuated OTA-induced EMT. Collagen content, determined by computerized analysis of Sirius red-stained kidney sections, increased in OTA, OTA-wine, and OTA-EtOH treated rats. In kidney cortex homogenates, COL-I and COL-III mRNA levels tended to rise in OTA treated rats, but were similar to CT after OTA-wine and OTA-E†OH administration. TIMP-1 gene expression was up-regulated in OTA, OTA-wine, and OTA-E†OH treated rats. LH2b mRNA/COL-I mRNA was significantly up-regulated in OTA-wine and OTA-E†OH treated rats, compared with CT and OTA alone. TGF- $\beta 1$ signaling tended to dominate after OTA, OTA-wine, and OTA-E†OH. MMP-1 protein levels were not affected. OTA induced proMMP-9 and $\alpha$ SMA overexpression, decreases of E-cadherin and N-cadherin, and DSC-2 up-regulation. OTA-wine caused a further, unexpected decrease of $\mathrm{E}$ - and $\mathrm{N}$-cadherins and further up-regulation of OTA-induced DSC-2, while strongly reducing the OTA-induced increases of $\alpha$ SMA and proMMP-9. Posttranslational collagen modifications, such as decreased collagen degradation through MMP inhibition and increased collagen cross-links, seem to be key mechanisms leading to OTA-induced kidney cortex fibrosis. This mechanism was not affected by red wine in these conditions. Red wine seems to have some protective role against OTA-induced EMT, although without completely blocking the process and determining a condition in which abundant cells display an intermediate translational phenotype, but there are no $\alpha$ SMA or epithelial markers.
\end{abstract}

Online address: http://molmed.org

doi: 10.2119/2005-00038.Gagliano

\section{INTRODUCTION}

Ochratoxin A (OTA) is a mycotoxin produced by some species of fungi such as Aspergillus and Penicillum. OTA is found as a contaminant of a variety of animal and human foods, including cereal and grain products, coffee, beer, and wine (1-3). OTA has been identified in blood, bile, and urine of humans and animals after consumption of contaminated food (4) and has been implicated as one of the etiological agents in Balkan endemic nephropathy, a chronic renal disease involving progressive renal fibrosis and impaired renal function, where contamination with much OTA is described (5). OTA is also immunosuppressive, teratogenic, genotoxic, and carcinogenic and affects blood coagulation and carbohydrate metabolism (6).

OTA-induced nephropathy is a progressive renal disease causing tubular degeneration, interstitial fibrosis, and impaired renal function (7). The toxin affects multiple sites of the nephron: acute exposure mainly affects the postproximal parts, whereas chronic exposure leads predominantly to damage of the proximal tubule (8), where uptake by tubular cells is achieved by an organic anion transport system (3). However, the exact mechanism of OTA toxicity on kidney has not yet been elucidated.

Light microscopy shows accumulation of the main extracellular matrix (ECM) components, particularly the collagen proteins, leading to glomerulosclerosis and tubulointerstitial fibrosis, a hallmark of various chronic renal diseases (9).

Collagen (COL) content reflects the dynamic balance between synthesis and degradation, with rapid turnover (10). Much of the newly synthesized COL is immediately degraded by matrix metalloproteinases (MMP), also present in the glomeruli and proximal tubules (11). MMP activity is closely regulated at several levels in cells, including the steps of synthesis and secretion and inactivation by specific inhibitors (TIMP). The overall balance of COL deposition can be affected by transforming growth factor- $\beta 1$

${ }^{1}$ Department of Human Morphology, University of Milan, Italy; ${ }^{2}$ Scientific Direction, Istituto Clinico Humanitas, Rozzano and "M. Rodriguez" Foundation, Institute for Quantitative Measures in Medicine, Milan, Italy; ${ }^{3}$ Department of Neuroscience (Pharmacology section) and Internal Medicine, University of Pisa, Italy. 
(TGF- $\beta 1)$ through a variety of biological actions: it directly increases the abundance and stability of COL-I and -III transcripts, but also reduces the synthesis of several MMPs (12) and increases the transcription of TIMP. The net effect of TGF- $\beta 1$ is to tip the balance of matrix turnover toward the accumulation of ECM, and in vitro and in vivo studies have convincingly shown that blocking TGF- $\beta 1$ suppresses COL production and subsequently inhibits the fibrotic process (13).

TGF- $\beta 1$ is antagonized by hepatocyte growth factor (HGF), a multifunctional peptide with antifibrotic action in various animal models (14). The balance between HGF and TGF- $\beta 1$ may be decisive in the pathogenesis of chronic renal fibrosis (15). To our knowledge there is still no information on how HGF is involved in the pathogenesis of OTA-induced kidney fibrosis.

Tubulointerstitial fibrosis is a common final pathway of chronic renal diseases leading to end-stage renal failure, irrespective of the nature of the initial renal injury. The process involves qualitative and quantitative changes in the composition of tubular basement membranes (TBMs), accumulation of myofibroblasts, and deposition of interstitial ECM components (16-18).

Activation of $\alpha$-smooth muscle actin ( $\alpha$ SMA)-positive myofibroblasts is considered a key event in the progression of renal fibrosis. Under pathologic conditions, tubular epithelial cells may transdifferentiate into myofibroblasts by a conversion known as tubular epithelial-mesenchymal transdifferentiation (EMT) (19).

EMT is a complex process in which renal tubular cells lose their polarized tubular epithelial phenotype and acquire new features characteristic of myofibroblasts, the major effector cells responsible for the excess deposition of interstitial ECM under pathologic conditions (20). Tubular epithelial cells lose E-cadherin, an adhesive junction protein expressed in differentiated and polarized epithelial cells, and acquire the myofibroblast marker $\alpha \mathrm{SMA}(21,22)$.

Gelatinases such as MMP-9 play a key role in promoting EMT, because disruption of the tubular basement membrane is important $(22,23)$. Accordingly, interstitial fibroblasts, responsible for the secretion of collagens that accumulate in tissue fibrosis, may come from tubular epithelial cells and can migrate in the interstitium and produce ECM proteins (24).

Using a morphological and molecular approach in an in vivo experimental model, this study was designed to characterize the effect of chronic OTA on the rat renal cortex, focusing on the analysis of fibrosis-related genes and proteins to help clarify the molecular mechanisms leading to OTA-induced kidney fibrosis. We particularly investigated COL content and the COL turnover pathway. Moreover, we analyzed the role of EMT in OTA-induced kidney fibrosis experimentally in vivo, investigating the key markers of EMT such as $\alpha \mathrm{SMA}$, E-cadherin, and proMMP-9, together with cytokines mainly involved in this mechanism. We also investigated, for the first time to our knowledge, the gene expression of desmosomal cadherins, as further possible markers of EMT leading to tubulointerstitial fibrosis in these experimental conditions.

The kidney is a site of intense oxidative processes and is extremely vulnerable to damage caused by free radicals. Oxidative stress has been suggested as one of the major causes of the toxicity of OTA $(25,26)$. Therefore, one might expect that the antioxidants in fruits, vegetables, tea, or wine would attenuate the renal injury caused by OTA. Both ethanol and antioxidant nonalcoholic wine components, mainly polyphenols, contained in red wine might counterbalance the OTA-induced free radicals $(27,28)$, so red wine could have some protective effect against OTAinduced kidney damage $(27,29)$.

In this study we also analyzed the effect of red wine on the overall molecular mechanisms leading to OTA-induced kidney fibrosis and whether a moderate intake of red wine influenced EMT in the kidney of OTA treated rats.

\section{MATERIALS AND METHODS}

\section{Animals}

A total of 32 male Wistar rats weighing 180 to $200 \mathrm{~g}$ were housed in single metabolic cages with controlled temperature $\left(25^{\circ} \mathrm{C}\right)$ and a 12-h alternating light-dark cycle. Rats were fed standard food and had free access to drinking water. All experiments were conducted in accordance with local ethics committee guidelines. Rats were divided into four groups of 8 , as follows.

Group 1, untreated controls (CT): each rat was given $0.5 \mathrm{~mL}$ saline by gastric gavage. Group 2, OTA: rats were treated for $90 \mathrm{~d}$ with a constant dose of OTA (Sigma Aldrich), $289 \mu \mathrm{g} / \mathrm{kg}$ every other day by gastric gavage, as previously reported (25). OTA was dissolved in $0.1 \mathrm{M} \mathrm{NaHCO}, \mathrm{pH} 7.4$, then diluted in $0.5 \mathrm{~mL}$ saline. Group 3, OTA-wine: rats were given OTA and red wine by gastric gavage. OTA was diluted in $0.5 \mathrm{~mL}$ of an OTA-free red wine (Barbera d'Asti, 13.5\%/vol). Group 4, OTA-EtOH: rats were given OTA diluted in $0.5 \mathrm{~mL}$ of an hydroalcoholic solution containing ethanol at the same concentration as that of red wine.

Finally, the animals were weighed and killed by an anesthetic overdose (chloralium hydrate), and the kidneys were removed. Kidney fragments containing both cortex and medulla were processed for morphological examination. For molecular analysis, the cortex was separated from the medulla and stored at $-80^{\circ} \mathrm{C}$. Gene and protein analysis was done on renal cortex homogenates.

\section{Histochemistry and Image Analysis}

Immediately after removal, kidney fragments were fixed in $4 \%$ formalin in $0.1 \mathrm{M} \mathrm{PBS}, \mathrm{pH} 7.4$, for $5 \mathrm{~h}$ at room temperature, routinely dehydrated, paraffin-embedded, and serially sectioned (thickness $5 \mathrm{Mm})$. Serial sections interspaced at $100 \mathrm{Mm}$ were stained with Sirius red and counterstained with hematoxylin; 5 sections were examined for each rat kidney. For Sirius red staining, slides were deparaffined and immersed for 30 minutes in saturated aqueous picric acid containing $0.1 \%$ Sirius red F3BA (Sigma, Milan, Italy), specific for COL, staining COL proteins distinctly red.

All the Sirius red-stained sections were analyzed by light microscopy, and the images were captured and digitized using an image analysis system with specific software (Isole, ICH, Italy). This software automatically selects the collagenous portion on the basis of similarities in the color of adjacent pixels, based on an RGB system. Tissue COL content is expressed by a fibrosis index $(\%)$ that indicates the ratio of the mean Sirius red-stained area to the mean whole area of the section, calculated as the mean of the fibrosis indexes for each section for each rat. 
Table 1. Primer sequences and RT-PCR amplification conditions.

\begin{tabular}{|c|c|c|c|c|}
\hline Gene & Primer sequence & $\begin{array}{c}\text { Annealing } \\
\text { temperature, }{ }^{\circ} \mathrm{C}\end{array}$ & $\begin{array}{l}\text { No. of } \\
\text { cycles }\end{array}$ & $\begin{array}{l}\text { Product } \\
\text { size, bp }\end{array}$ \\
\hline \multirow[t]{2}{*}{ COL-I } & 5'-TTGACCCTAACCAAGGATGC & 54 & 30 & 197 \\
\hline & 5'-CACCCCTTCTGCGTTGTATT & & & \\
\hline \multirow[t]{2}{*}{ COL-III } & 5'-AGGCCAATGGCAATGTAAAG & 53 & 30 & 187 \\
\hline & 5'-GGCCTTGCGTGITTGATATT & & & \\
\hline \multirow[t]{2}{*}{ TGF- $\beta 1$} & 5'-CACCTGCACAGCTCCAGGCAC & 54 & 32 & 153 \\
\hline & 5'-CTTGCGACCCACGTAGTAGACG & & & \\
\hline \multirow[t]{2}{*}{ HGF } & 5'-TCGTTCCTTGGGATTATTGC & 55 & 32 & 571 \\
\hline & 5'-TGGTGCTGACTGCATTTCTC & & & \\
\hline \multirow[t]{2}{*}{ TIMP-1 } & 5'-TCCCCAGAAATCATCGAGAC & 55 & 32 & 329 \\
\hline & 5'-ATGGCTGAACAGGGAAACAC & & & \\
\hline \multirow[t]{2}{*}{ LH2b } & 5'-TGCGATCAGAGATGAGTGAA & 53 & 32 & 229 \\
\hline & 5'-GTTGAGATGGGAAGTGTTGTAAT & & & \\
\hline \multirow[t]{2}{*}{ E-cadherin } & 5'-GCAGTTCTGCCAGAGAAACC & 55 & 28 & 315 \\
\hline & 5'- AATCCTGCTTCCAGGGAGAT & & & \\
\hline \multirow[t]{2}{*}{ N-cadherin } & 5'-TGTTGCTGCAGAAAACCAAG & 53 & 28 & 309 \\
\hline & 5'-GGCGACTCTCTGTCCAGAAC & & & \\
\hline \multirow[t]{2}{*}{ DSG-2 } & 5'-CCTTGGTGGACCAGCACTAT & 57 & 35 & 316 \\
\hline & 5'-GACAGCACCGTCTTCCTAGC & & & \\
\hline \multirow[t]{2}{*}{ DSC-2 } & 5'-CGTAATTCCCGTCCGAGTTA & 55 & 35 & 386 \\
\hline & 5'-TTGAGCTGTGAAGCCATTTG & & & \\
\hline \multirow[t]{2}{*}{ GAPDH } & 5'-ATGGTGAAGGTCGGTGTGAAC & 54 & 28 & 473 \\
\hline & 5'- GCTGACAATCTTGAGGGAGT & & & \\
\hline
\end{tabular}

\section{RT-PCR}

Total RNA was extracted from approximately $100 \mathrm{mg}$ frozen renal cortex by a modification of the acid guanidinium thiocyanate-phenolchloroform method (Tri-Reagent; Sigma). RNA purity and concentration were determined spectrophotometrically. One microgram total RNA was digested with DNase I then reverse-transcribed in $20 \mathrm{ML}$ final volume of reaction mix (Promega Italia, Milan, Italy).

The primers used and protocols for RT-PCR are listed in Table 1. Amplification reactions were conducted in a final volume of $25 \mathrm{ML}$ containing $2.5 \mathrm{ML}$ cDNA, $200 \mathrm{MM}$ of the $4 \mathrm{dNTPs}, 100 \mathrm{pmol}$ of each primer, and 2.5 units Taq DNA polymerase (EuroTaq, Euroclone, Pero, Milan, Italy).

The RT-PCR products were electrophoresed on $1.5 \%$ agarose gels, stained with ethidium bromide, and quantified by densitometric analysis (Image Pro-Plus). The results were normalized on GAPDH gene expression.

\section{Protein Analysis}

Renal cortex samples were homogenized in ice-cold extraction buffer (1 mL/100 mg tissue) containing Tris- $\mathrm{HCl} 50 \mathrm{mM}, \mathrm{pH}$ 7.5, $\mathrm{NaCl} 100 \mathrm{mM}$, and $\mathrm{CaCl}_{2} 2 \mathrm{mM}$. The homogenates were centrifuged $\left(4^{\circ} \mathrm{C}, 5 \mathrm{~min}, 14,000 \mathrm{~g}\right)$, and the supernatant was decanted and saved on ice. The final concentration of the renal cortex extracts was determined with a standardized colorimetric assay (DC Protein Assay; Bio-Rad), and the samples were divided into aliquots and stored at $-20^{\circ} \mathrm{C}$.

Dot blot and immunoassay. Total protein extracts $(25 \mathrm{mg}$ for each sample in a final volume of $200 \mathrm{~mL}$ TBS) were spotted in triplicate on a nitrocellulose membrane in a Bio-Dot SF apparatus
(Bio-Rad). After the entire sample had filtered through the membrane under gentle vacuum, each sample well was washed with 300 ML TBS. After complete draining, the membrane was air-dried for $30 \mathrm{~min}$ then placed in blocking solution for $1 \mathrm{~h}$.

For MMP-1 determination, the membrane reacted with a monoclonal antibody to MMP-1 $(0.5 \mathrm{mg} / \mathrm{mL}$ in PBST/BSA $1 \% / \mathrm{NaN}_{3} 0.02 \%$ ) (Ab-1; Calbiochem) for $1 \mathrm{~h}$ and with a HRPconjugated rabbit anti-mouse serum (Sigma); the immunoreactive bands were revealed by Amplified Opti-4CN (Bio-Rad) and scanned densitometrically.

SDS-zymography. Renal cortex extracts were thawed on ice and mixed 3:1 with substrate gel sample buffer (10\% SDS, 4\% sucrose, $0.25 \mathrm{M}$ Tris- $\mathrm{HCl}$, pH 6.8, 0.1\% bromophenol blue). Each sample $(30 \mathrm{Mg})$ was loaded under nonreducing conditions onto electrophoretic minigels (SDS-PAGE) containing $1 \mathrm{mg} / \mathrm{mL}$ of type I gelatin (Sigma). The gels were run at $15 \mathrm{~mA} /$ gel through the stacking phase $(4 \%)$ and at $20 \mathrm{~m} \AA /$ gel for the separating phase $(10 \%)$, with a running buffer temperature of $4^{\circ} \mathrm{C}$. After SDSPAGE, the gels were washed twice in $2.5 \%$ Triton X-100 for $30 \mathrm{~min}$ each, rinsed in water, and incubated overnight in a substrate buffer (Tris- $\mathrm{HCl} 50 \mathrm{mM}, \mathrm{CaCl}_{2} 5 \mathrm{mM}, \mathrm{NaN}_{3} 0.02 \%, \mathrm{pH} 8$ ) at $37^{\circ} \mathrm{C}$. The MMP gelatinolytic activity was detected after staining the gels with Coomassie brilliant blue R250, as clear bands on a blue background. To confirm the identity of this activity, purified MMP-1 and MMP-2 (100 ng) (Calbiochem) were run as standards.

Western blot. For each sample $25 \mathrm{mg}$ total protein extracts were diluted in SDS sample buffer, loaded on 10\% SDS polyacrylamide gel, separated under reducing and denaturing conditions at $80 \mathrm{~V}$ according to Laemmli (30), and transferred at $90 \mathrm{~V}$ to a nitro- 
Table 2. Mean body weight (BW) and kidney weight (KW) and ratio of $\mathrm{KW}$ to $\mathrm{BW}$ in CT, OTA, OTA-wine, and OTA-EtOH treated rats.

\begin{tabular}{llll} 
Treatment & BW, g & KW, g & KW:BW \\
\hline CT & 412.5 & 1.02 & 0.0025 \\
OTA & $355.0^{a}$ & $0.87^{a}$ & 0.0024 \\
OTA-Wine & $405.8^{b}$ & $0.96^{a}$ & 0.0024 \\
OTA-E†OH & 381.0 & $0.86^{a}$ & 0.0023
\end{tabular}

ap $<0.05$ vs. CT; ${ }^{b} P<0.05$ vs. OTA-wine.

cellulose membrane in $0.025 \mathrm{M}$ Tris, $192 \mathrm{mM}$ glycine, and 20\% methanol, $\mathrm{pH}$ 8.3. After electroblotting, the membranes were airdried and blocked for 1 hour. After being washed in TBST (TBS/ Tween-20 0.05\%), membranes were incubated for $1 \mathrm{~h}$ at room temperature in monoclonal antibody to $\alpha$ SMA (1:500 in TBST) (clone 1A4; Sigma) and, after washing again, in HRP-conjugated rabbit anti-mouse serum (1:80,000 dilution; Sigma). Immunoreactive bands were revealed using the Opti-4CN substrate (Bio-Rad).
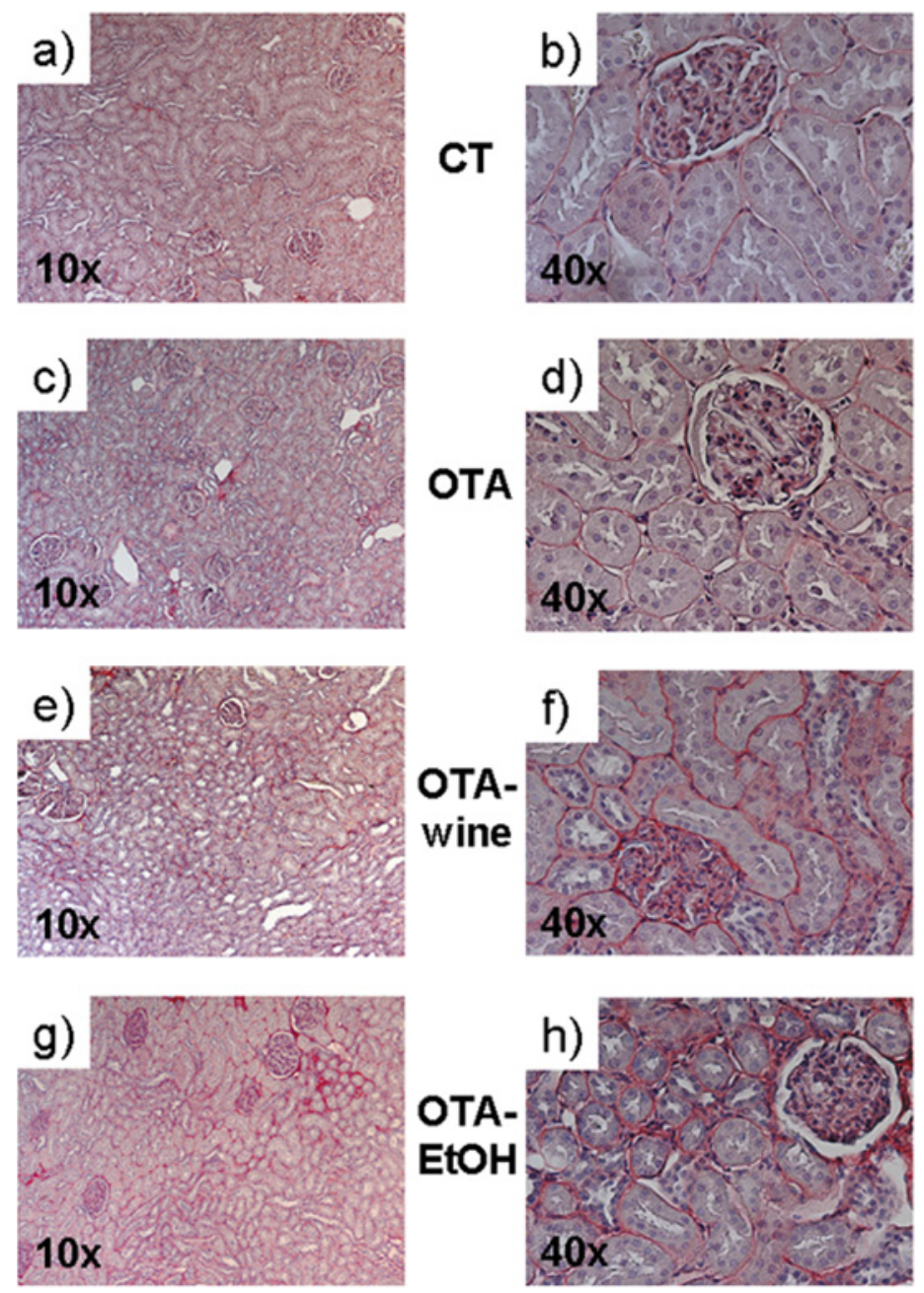

Figure 1. Microphotographs of Sirius red-stained kidney sections of CT (a, b), OTA (c, d), OTA-wine $(e, f)$, and OTA-EłOH $(g, h)$ treated rats. Original magnification $\times 10(a, c, e, g)$ and $\times 40(b, d, f, h)$.

\section{fibrosis index (\%)}

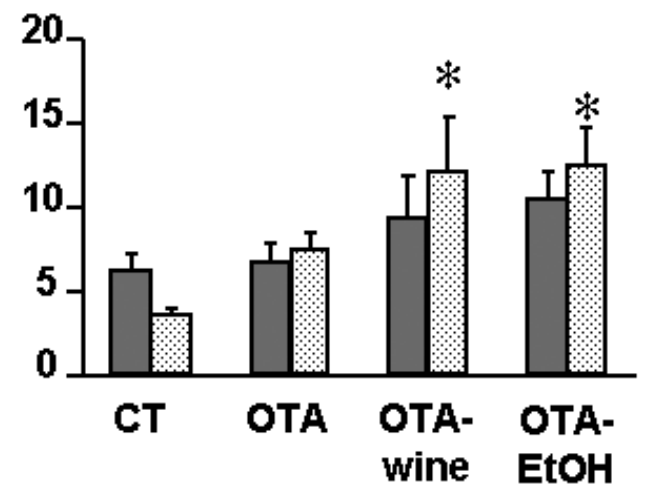

$\square$ kidney kidney cortex

Figure 2. Bar graphs showing the fibrosis index obtained by computerized analysis of kidney sections of CT, OTA, OTA-wine, and OTA-E†OH treated rats. The index indicates tissue collagen content and is calculated as described in "Materials and Methods." Means \pm SEM. ${ }^{*} P<0.05$ compared with CT renal cortex.

\section{Statistical Analysis}

Experimental groups were compared by one-way ANOVA, followed by the Student-Neumann-Keuls post test. Results are expressed as mean \pm SEM. A $P$ value less than 0.05 was considered significant.

\section{RESULTS}

\section{Kidney and Body Mass}

The kidney weights (KW) and body mass (BW) of CT and rats treated with OTA, OTA-wine, and OTA-EtOH are presented in Table 2. Neither treatment had any effect on kidney weight, and the ratio of $\mathrm{KW}$ to $\mathrm{BW}$ was similar in all groups.

\section{Morphological and Quantitative Image Analysis}

Light microscopy analysis of Sirius red-stained paraffin-embedded rat kidney sections indicated diffuse fibrosis in the whole kidney of rats in all treatment groups, compared with CT. COL accumulation was evident in the tubulointerstitium, but the glomeruli did not seem to be affected (Figure 1).

COL content, expressed as the fibrosis index, rose in the whole kidney of OTA, OTA-wine, and OTA-EtOH treated rats, compared with $\mathrm{CT}$, but more in the cortex; the fibrosis index was high in the cortex of all treated groups $(116 \%, \mathrm{NS} ; 244 \%, P<0.05$; $255 \%, P<0.05$ vs. CT; ANOVA $P=0.015$ ) (Figure 2).

Expression of Fibrosis-Related Genes in the Renal Cortex The changes in the abundance of COL-I, COL-III, TIMP-1, and LH2b transcripts in renal cortex homogenates are presented in Figure 3. 


\section{a)}

COL-I MRNA

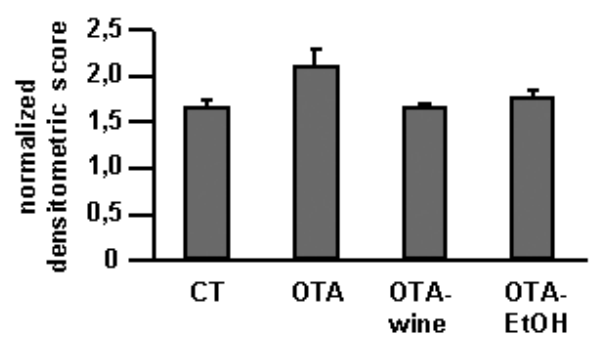

b) COL-III mRNA

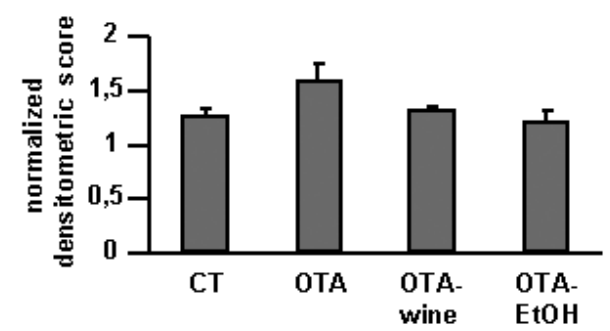

c)

TIMP-1 mRNA

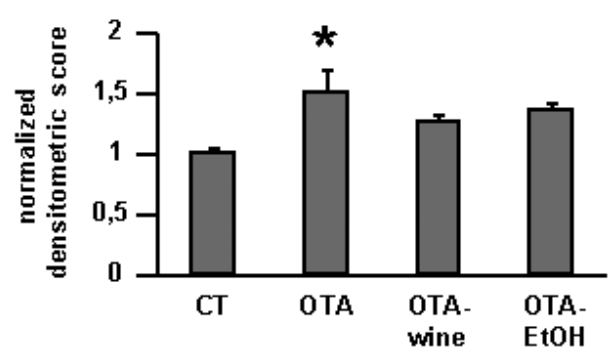

$\begin{array}{lll}\text { d) LH2b mRNA } & \text { e) LH2b/COL-I mRNA }\end{array}$
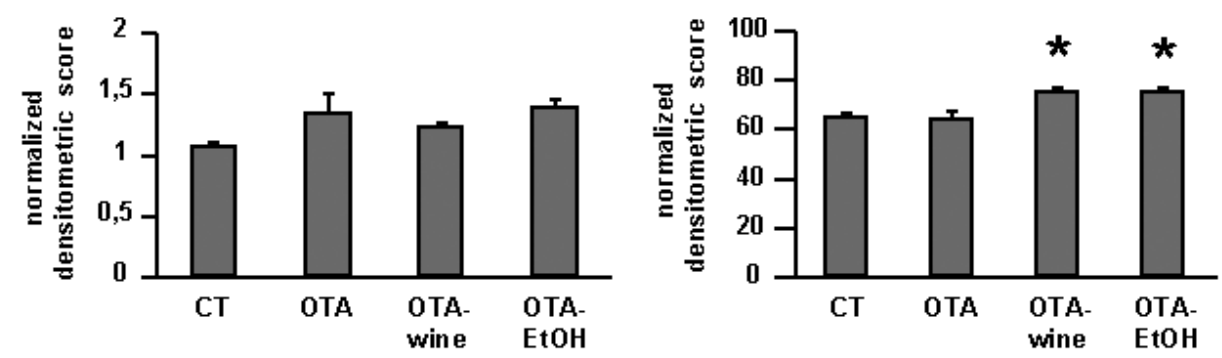

Figure 3. Bar graphs showing COL-I (a), COL-III (b), TIMP-1 (c), LH2b (d), and LH2/COL-I (e) mRNA levels in CT and OTA treated rats. Changes in mRNA are expressed as normalized densitometric units relative to GAPDH mRNA. Means \pm SEM. ${ }^{*} P<0.05$ compared with $C T$.

OTA raised COL-I mRNA levels by $25 \%$ compared with CT. In OTA-wine and OTA-EtOH treated animals, COL-I gene expression was similar to CT (ANOVA $P=0.060$ ) (Figure 3a). COL-III mRNA levels showed a similar pattern, with a $26 \%$ increase in OTA treated rats compared with CT and no effect on gene expression in OTA-wine and OTA-EtOH treated animals (ANOVA $P=0.087$ ) (Figure $3 b$ ). TIMP-1 gene expression was up-regulated in OTA, OTA-wine, and OTA-EtOH treated rats (respectively, by 51\% $P<0.05 ; 27 \%$, NS; and 36\%, NS compared with CT; ANOVA $P=0.029$ ) (Figure 3c). LH2b gene expression tended to be higher in OTA, OTA-wine, and OTA$\mathrm{EtOH}$ treated rats than in CT (respectively, 26\%, 15\%, and 30\%,
NS compared with CT) (Figure 3d). If LH2b mRNA levels are expressed in relation to COL-I mRNA levels, OTA-wine (116\% and $117 \%, P<0.05$ compared with CT and OTA treated) and OTA-EtOH $(116 \%, P<0.05$ vs. CT) rats had the highest LH2b gene expression (Figure $3 \mathrm{e}$ ).

For TGF- $\beta 1$ gene expression, as described for interstitial COL, TGF- $\beta 1$ mRNA levels were $24 \%$ and $31 \%$ higher, respectively, in OTA and OTA-EtOH treated rats than in CT (NS); in OTA-wine treated animals, TGF- $\beta 1$ gene expression was similar to that in CT (Figure 4a).

HGF gene expression was slightly affected by OTA with or without wine or EtOH (Figure 4b). If we consider the ratio TGF- 
a)

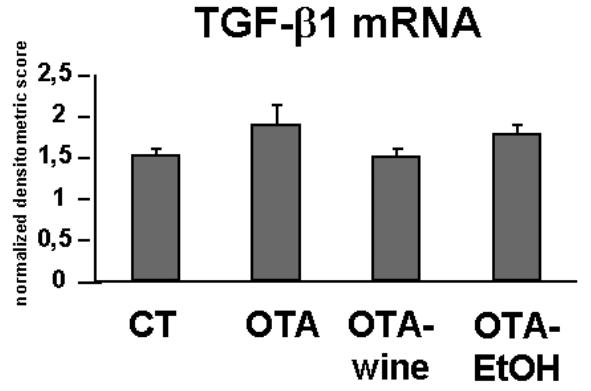

b)

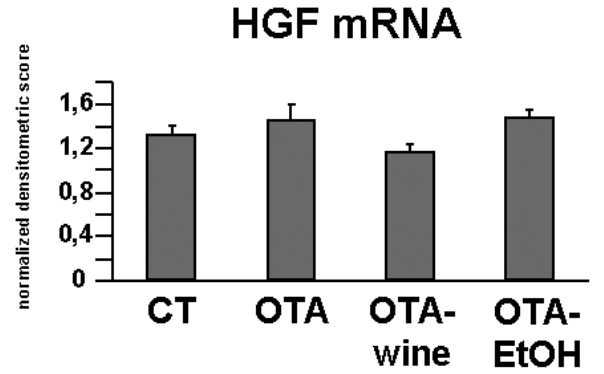

c)

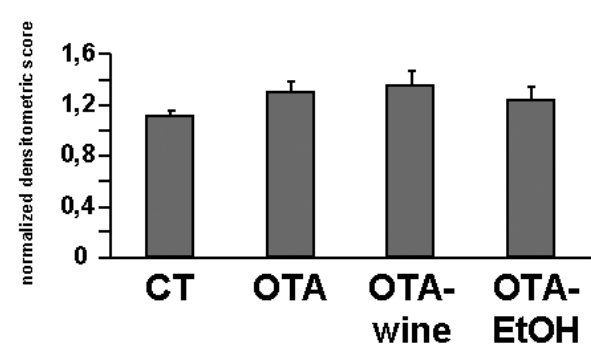

Figure 4. Bar graphs showing TGF- $\beta 1$ (a), HGF (b) mRNA levels and the ratio of TGF- $\beta 1$ to HGF mRNA (c) in CT, OTA, OTA-wine and OTA-EtOH treated rats. Changes in mRNA are expressed as normalized densitometric units relative to GAPDH mRNA. Means \pm SEM.

$\beta 1$ /HGF, TGF- $\beta 1$ signaling tended to dominate after OTA alone $(16 \%, \mathrm{NS})$ and with wine $(20 \%, \mathrm{NS})$ or EtOH $(11 \%, \mathrm{NS})$ (Figure $4 \mathrm{c})$.

\section{Collagen Degradation}

MMP-1 protein levels were evaluated by dot blot. The antibody recognized both the latent and active forms of interstitial collagenase. Densitometric analysis of MMP-1-immunoreactive bands indicated that interstitial collagenase expression was similar in renal cortex homogenates from all the experimental groups (Figure 5).

\section{E- and N-Cadherins and DSG-2 and DSC-2 Gene Expression}

E-cadherin mRNA levels dropped 7\% after OTA (NS), 23\% after OTA plus wine $(P<0.05$ compared with CT and OTA), and $8 \%$ after OTA plus EtOH (NS) (Figure 6a). N-cadherin gene expression gave a similar pattern, though without significant differences (Figure 6b). DSG-2 gene expression was similar in all experimental groups (data not shown). DSC-2 mRNA levels, however, increased steeply after OTA, OTA-wine, and OTA-EtOH (respectively, 40\%, 45\%, and $22 \%, P<0.05$ compared with CT) (Figure 6c).

\section{MMP-1 protein levels}

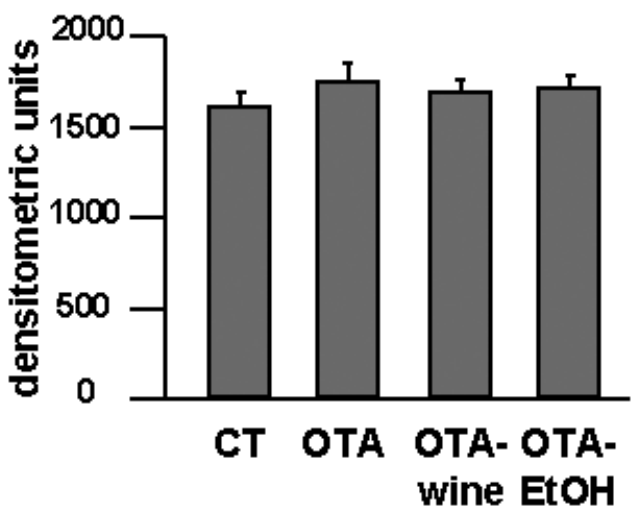

Figure 5. Bar graphs showing MMP-1 protein levels after densitometric analysis of immunoreactive and lysis bands obtained by dot blot.

\section{ProMMP-9 Protein Levels}

SDS-zymography showed lysis bands weighing $92 \mathrm{kDa}$, consistent with proMMP-9, and lysis bands of higher molecular weight consistent with MMP-9 polymers. The densitometric analysis of bands consistent with gelatinase B indicated, respectively, a 59\% increase $(P<0.05$ compared with $C T)$ and a $52 \%$ decrease $(P<0.05$ compared with OTA) in OTA and OTA-wine treated rats compared with $\mathrm{CT}$, and a $152 \%$ increase in OTA-EtOH treated rats compared with $\mathrm{CT}(P<0.05$ compared with $\mathrm{CT}$, OTA, and OTAwine) (Figure $7 \mathrm{a}, \mathrm{b}$ ).

\section{$\alpha$ SMA Protein Expression}

$\alpha$ SMA protein expression was evaluated by Western blot. Densitometric analysis of immunoreactive bands showed much stronger expression $(95 \%)$ in OTA treated rats compared with CT. After OTA-wine treatment, $\alpha$ SMA protein expression fell to CT levels, $66 \%$ lower than in OTA treated rats $(P<0.05)$. Also, after OTA$\mathrm{EtOH}$ treatment, $\alpha \mathrm{SMA}$ protein expression was similar to $\mathrm{CT}$ levels (Figure 8a, b).

\section{DISCUSSION}

Because there is some evidence of a correlation between the presence of OTA in human blood and food samples and the incidence of human nephropathies (31), and OTA induces tubulo-interstitial nephropathy in animals similar to the human variety $(4,32)$, we investigated the overall molecular mechanisms leading to the ECM remodeling in the kidney of OTA treated rats, and the possible protective role of red wine on this injured kidney.

\section{OTA and Kidney Fibrosis}

Renal fibrosis is characterized by changes of tubular basement membrane and interstitial matrix, tubular atrophy, and the accumulation of myofibroblasts $(17,18,33)$.

The analysis of renal cortex COL content found strong cortical fibrosis in OTA treated rats. Enhanced COL deposition in the kidney may result from reduced breakdown by proteolytic 
a)

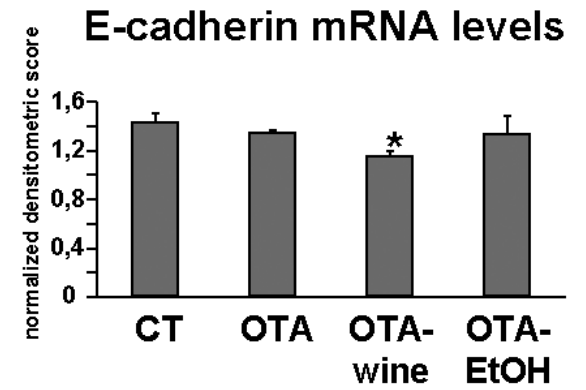

b)

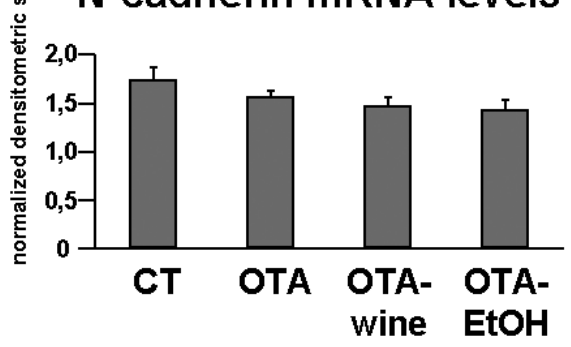

c)

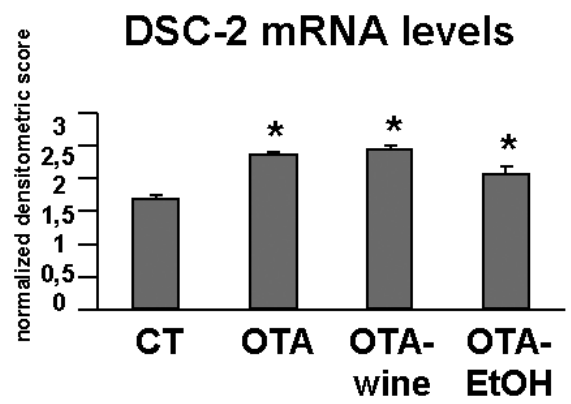

Figure 6. Bar graphs showing E-cadherin (a), N-cadherin (b), and DSC-2 (c) gene expression in CT, OTA, OTA-wine, and OTA-EtOH treated rats. Changes in mRNA are expressed as normalized densitometric units relative to GAPDH mRNA. Means \pm SEM. ${ }^{*} P<0.05$ compared with CT.

enzymes such as MMP, whose activity is closely regulated; any mismatch in pathological conditions could result in excessive ECM accumulation (34-36).

We found MMP-1 levels in the renal cortex of OTA, OTA-wine, and OTA-EtOH treated rats similar to those in CT, suggesting that the interstitial COL accumulation indicated by Sirius red staining is not a consequence of lower levels of interstitial collagenase. This is in line with the results on TIMP- 1 and LH2b gene expression.

TIMP-1 mRNA levels were significantly up-regulated by OTA, and tended to increase after OTA-wine and OTA-EtOH treatment, compared with CT. Because TIMP-1 inhibits active MMP-1 in a 1:1 stoichiometric relationship, its overexpression in OTA, OTA-wine, and OTA-EtOH treated rats, though showing similar interstitial collagenase expression, suggests that MMP-1 inhibition may be one of the molecular mechanisms underlying COL accumulation in OTA and OTA-wine treated rats, leading to the histological pattern expressed by the fibrosis index. In particular, TIMP-1 up-regulation in OTA treated rats is consistent with the increased kidney cortex collagen deposition.

This result is borne out by LH2b gene expression. We analyzed the long LH2b (37) gene expression whose increase is a gen- a) proMMP-9 protein levels

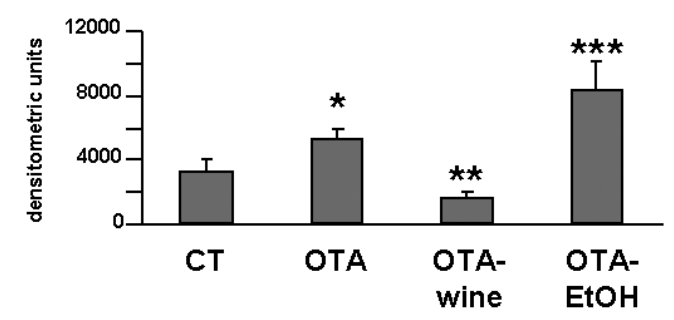

b)

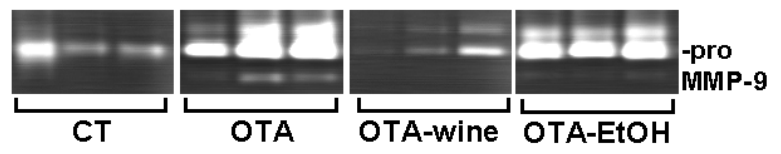

Figure 7. (a) Bar graph showing proMMP-9 levels after densitometric analysis of immunoreactive and lysis bands obtained by SDS-zymography (means \pm SEM). (b) Representative gelatin zymogram of MMP in kidney cortex homogenates of CT, OTA, OTA-wine, and OTA-E†OH treated rats. The lysis bands are consistent with proMMP-9. Each lane represents a single rat. ${ }^{*} P<0.05$ compared with $C T$; ${ }^{* *} P<0.05$ compared with OTA; ${ }^{* * *} P<0.05$ vs. OTA, CT, OTA-wine.

eral fibrotic phenomenon leading to the over-hydroxylation of the COL telopeptides, contributing to unwanted $\mathrm{COL}$ accumulation (38). The LH2b/COL-I mRNA ratio indicates the gene expression of LH2b relative to COL-I. This ratio was significantly higher in OTA-wine and OTA-EtOH treated rats than in the CT and OTA groups, suggesting that LH2b induction exceeds that of COL-I and that the synthesized COL will become over-hydroxylated (38). Because cross-linked COL is harder to degrade, this is

a) $\alpha$ SMA protein levels

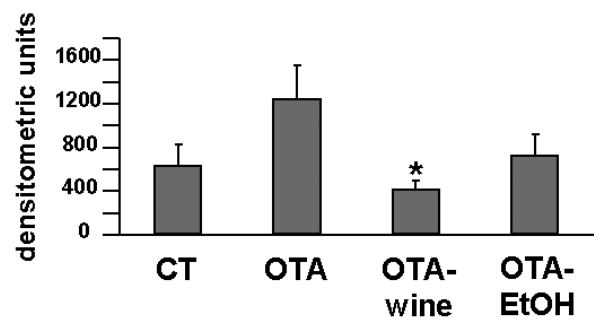

b)

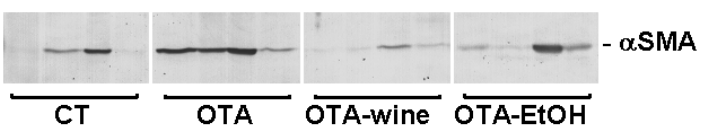

Figure 8. (a) Bar graph showing $\alpha$ SMA protein levels after densitometric analysis of immunoreactive obtained by western blot (means \pm SEM). (b) Representative Western blot of $\alpha S M A$ in kidney cortex homogenates of CT, OTA, OTA-wine, and OTA-E†OH treated rats. The immunoreactive band is $\alpha$ SMA. Each lane represents a single rat. ${ }^{*} P<0.05$ compared with OTA. 
another mechanism helping to explain why renal cortex tubulointerstitial fibrosis is more evident in OTA-wine and OTA-EtOH treated rats, according to the results of the fibrosis index.

Pivotal cytokines such as HGF and TGF- $\beta 1$ are very likely involved in this mechanism. HGF has antifibrotic effects, including blockade of EMT $(14,24)$. Conversely, TGF- $\beta 1$ is the major isoform involved in fibrosis; not only does it affect the formation of connective tissue, but it is also probably the most important mediator of the EMT.

HGF and TGF- $\beta 1$ gene expressions were slightly affected by OTA with or without wine or ethanol, suggesting that OTA itself induces a response in injured kidneys. However, the TGF- $\beta 1 /$ HGF mRNA ratio, as previously described $(14,39)$, favors TGF- $\beta 1$, so in OTA and to a greater extent in OTA-wine and OTA-EtOH treated rats, the ratio tended to be out of balance and TGF- $\beta 1$ signaling dominated. This is consistent with our findings on renal cortex COL content and TIMP-1 gene expression, confirming this cytokine as a key mediator in fibrosis, although with no significant differences in control and treated rats. It is conceivable that the chronic OTA schedule we used, with longer treatment, might well have worthwhile activity in the mechanisms leading to OTAinduced kidney injury.

\section{OTA and EMT}

Epidemiological findings indicate that moderate consumption of wine is associated with lower mortality from cardiovascular and cerebrovascular diseases (40), but the kidney might also be one of the organs enjoying a wine-induced protective effect, because reactive oxygen species play a key role in the mechanisms of seemingly unrelated nephropathies (27).

Oxidative stress may well be a component of the toxicity of OTA $(25,26)$, and antioxidant compounds such as melatonin have been reported to counteract oxidative stress in rats fed an OTAcontaminated diet $(41,42)$.

We analyzed the molecular mechanisms of EMT and the possible protective effect of red wine on rat OTA-injured kidney.

The key role of EMT, especially in the early phases of OTAinduced renal fibrosis, is supported by recent findings (43) that OTA uptake occurs in tubular epithelial cells, but not in fibroblasts because they have no transporter for OTA.

To make EMT possible, changes in the expression of various genes accomplish epithelial-to-mesenchymal phenotypic conversion, and cellular events lead to the completion of the process.

A loss of E-cadherin is one of the features of EMT, together with the expression of the myofibroblast marker $\alpha \mathrm{SMA}$ and the disruption of TBM by gelatinases, an event of pivotal importance in clearing the path for transformed cells to migrate toward the interstitium $(44,45)$.

We found that OTA induced a tendency to decrease for Ecadherin and N-cadherin, the 2 prototypical cadherins of the tubular epithelial cell phenotype (46). Because tubular epithelial cells express desmosomes containing the cadherins DSC-2 and DSG-2, we investigated, for the first time to our knowledge, whether OTA also influences the expression of these epithelial markers. Whereas DSG-2 was unaffected, DSC-2 gene expression was strongly up-regulated by OTA. Interestingly, OTA-wine and
OTA-EtOH caused a further, unexpected decrease of the E- and $\mathrm{N}$-cadherins, and OTA further up-regulated DSC-2. By contrast, red wine strongly reduced the OTA-induced increase of $\alpha \mathrm{SMA}$ and proMMP-9 protein levels, that, by contrast, remained highly expressed after OTA-EtOH.

These results point to an effect on the mechanisms of EMT in OTA-wine treated rats and suggest that the nonalcoholic components of red wine exert a protective role on OTA-induced EMT, reducing migration of epithelial converted cells into the interstitium and favoring the transition of myofibroblasts to the epithelial phenotype.

However, red wine did not block OTA-induced E- and Ncadherin down-regulation. Possibly the wine prevents the progress of tubular epithelial cells through a mesenchymal-toepithelial transitional stage, so they lose the myofibroblast marker $\alpha S M A$. The unexpected concomitant loss of the tubular markers Eand N-cadherin suggests a condition in which there are abundant cells at the translational stage with absence of $\alpha$ SMA and tubular markers, thus displaying an intermediate translational phenotype.

EMT is a highly dynamic process with several intermediate stages (45), and our findings are consistent with the observation that several cells are still positive for epithelial markers in the widened interstitium of end-stage diseased kidneys in patients during EMT (47). Because tubular epithelial cells are developmentally derived from the metanephrogenic mesenchyme through mesenchymal-to-epithelial conversion (48), hypothetically red wine might induce a similar transition, preserving the tubular epithelial phenotype and blocking OTA-induced EMT.

There is very little information on desmosomal cadherins DSC-2 and DSG-2 in the kidney in physiological or pathologic conditions, both of which play a role in directing differentiation in epithelial tissues (49). Therefore they may be needed during EMT.

DSC-2 was strongly up-regulated after OTA, OTA-wine, and OTA-EtOH. On the basis of a previous suggestion that DSC-2 might act as a limiting factor for desmosome assembly during differentiation (50), we can speculate that OTA may help reduce cell adhesion between tubular epithelial cells and that red wine did not affect this.

The overall EMT process is governed by cytokines such as TGF- $\beta 1$ and HGF $(14,51)$. Our data show that OTA and, to a greater extent, OTA-wine and OTA-EtOH, shifts the TGF- $\beta 1 /$ HGF ratio toward TGF- $\beta 1$, suggesting that this cytokine is involved in the events leading to OTA-induced EMT and that red wine is not able to balance the TGF- $\beta 1 /$ HGF ratio.

\section{Conclusions}

As a whole, our data point to EMT as a key event in the development of renal cortical fibrosis triggered by OTA. In these experimental conditions, red wine protects the kidney, somehow inhibiting the molecular mechanisms of this transition. This effect is very likely mediated by the nonalcoholic components of red wine. After chronic treatment, however, OTA induces evident tubulointerstitial fibrosis. Posttranslational COL modifications, such as reduced COL degradation through inhibition of MMP-1 by TIMP-1 and increased COL cross-links, are the key mechanisms leading to OTA-induced renal cortical fibrosis. This mecha- 
nism very likely predominated and was unaffected by red wine in these experimental conditions.

Altogether, these data give new insights on the description of the molecular events triggered by OTA dietary intake in kidney and may be useful to understand the interactions between the toxin and nutrients currently assumed by dietary intake, and therefore contribute to the assessment of the human health risk posed by OTA.

\section{ACKNOWLEDGMENTS}

We thank Silvia Celon and Osvaldo De Negri for their technical support and J.D. Baggott for editing the text. This work was supported by a grant of the Italian Government (Ministero delle Politiche Agricole e Forestali, MPAF) Targeted Project.

Address correspondence and reprint requests to Nicoletta Gagliano, Department of Human Morphology - LITA Segrate, Via Fratelli Cervi 93, 20090 Segrate, Milano, Italy. Phone: +39.02 .50330462 ; fax: +39.02 . 50330452; e-mail: nicoletta.gagliano@unimi.it.

Submitted November 21, 2005; accepted for publication March 6, 2006.

\section{REFERENCES}

1. Marquardt RR, Frohlich A. (1992) A review of recent advances in understanding ochratoxicosis. J. Anim. Sci. 70:3968-88

2. Walker R. (2002) Risk assessment of ochratoxin: current views of the European Scientific Committee on Food, the JECFA and the Codex Committee on Food Additives and Contaminants. Adv. Exp. Med. Biol. 504:249-55.

3. O'Brien E, Dietrich DR. (2005) Ochratoxin A: the continuing enigma. Crit. Rev. Toxicol. 35:33-60.

4. Petkova-Bocharova T, Chernozemski IN, Castegnaro M. (1988) Ochratoxin A in human blood in relation to Balkan endemic nephropathy and urinary system tumours in Bulgaria. Food Addit. Contam. 5:299-301.

5. Vrabcheva T et al. (2004) Analysis of ochratoxin A in foods consumed by inhabitants from an area with Balkan endemic nephropathy: a 1 month follow-up study. J. Agric. Food. Chem. 52:2404-10.

6. Pfohl-Leszkowicz A, Petkova-Bocharova T, Chermozemsky IN, Castegnaro $M$. (2002) Balkan endemic nephropathy and associated urinary tract tumours: a review on aetiological causes and the potential role of mycotoxins. Food. Addit. Contam. 19:282-302.

7. Krogh P. (1992) Role of ochratoxin in disease causation. Food. Chem. Toxicol. 30:213-24.

8. Gekle M, Silbernagl S. (1996) Renal toxicodynamics of ochratoxin A: a pathophysiological approach. Kidney Blood Press. Res. 19:225-35.

9. Klahr S, Schreiner G, Ichikawa I. (1988) The progression of renal disease. N. Engl. J. Med. 318:1657-66.

10. Laurent GJ. (1987) Dynamic state of collagen degradation in vivo and their possible role in regulation of collagen mass. Am. J. Physiol. 252: C1-9.

11. Lovett DH, Sterzel RB, Kashgarian M, Ryan JL. (1983) Neutral proteinases activity produced in vitro by cells of the glomerular mesangium. Kidney Int. 23:342-9.

12. Sharma K, Ziyadeh FN. (1994) The emerging role of transforming growth factorbeta in kidney diseases (editorial). Am. J. Physiol. 266:F829-42.

13. Basile DP. (2001) Transforming growth factor-beta as a target for treatment in diabetic nephropathy. Am. J.Kidney Dis. 38:887-92.

14. Liu Y. (2004) Hepatocyte growth factor in kidney fibrosis: therapeutic potential and mechanisms of action. Am. J. Physiol. Renal. Physiol. 287:F7-16.

15. Mizuno S et al. (2000) Reciprocal balance of hepatocyte growth factor and transforming growth factor-beta 1 in renal fibrosis in mice. Kidney Int. 57: 937-48.

16. Eddy AA. (1996) Molecular insights into renal interstitial fibrosis. J. Am. Soc. Nephrol. 7:2495-508

17. Remuzzi G, Bertani T. (1998) Pathophysiology of progressive nephopathies. N. Engl. J. Med. 339:1448-56.

18. Abrass CK et al. (1999) Unique changes in interstitial extracellular matrix composition are associated with rejection and cyclosporine toxicity in human renal allograft biopsies. Am. J. Kidney Dis. 33:11-20.
19. Okada H, Danoff TM, Kalluri R, Neilson EG. (1997) Early role for Fspl in epithelialmesenchymal transformation. Am. J. Physiol. 273:F563-74.

20. Powell DW et al. (1999) Myofibroblasts. I. Paracrine cells important in health and disease. Am. J. Physiol. 277:C1-9.

21. Yang J, Lui J. (2002) Blockage of epithelial to myofibroblast transition by hepatocyte growth factor prevents renal interstitial fibrosis. J. Am. Soc. Nephrol. 13:96-107.

22. Yang J, Liu Y. (2001) Dissection of key events in tubular epithelial to myofibroblast transition and its implications in renal interstitial fibrosis. Am. J. Pathol. 159: 1465-75.

23. Cheng S, Lovett DH. (2003) Gelatinase A (MMP-2) is necessary and sufficient for renal tubular cell epithelial-mesenchymal transformation. Am. J. Pathol. 162: 1937-49.

24. Liu Y. (2005) Epithelial to mesenchymal transition in renal fibrogenesis: pathologic significance, molecular mechanisms, and therapeutic intervention. J. Am Soc. Nephrol. 15:1-12.

25. Baudrimont I et al. (1994) Effect of superoxide dismutase and catalase on the nephrotoxicity induced by subchronical administration of ochratoxin A in rats. Toxicology 89:101-11.

26. Schaaf GJ et al. (2002) The role of oxidative stress in the ochratoxin A-mediated toxicity in proximal tubular cells. Biochim. Biophys. Acta 1588:149-58.

27. Rodrigo R, Rivera GR. (2002) Renal damage mediated by oxidative stress: a hypothesis of protective effects of red wine. Free Rad. Biol. Med. 33:409-22

28. Rodrigo R et al. (2002) Rat kidney antioxidant response to long-term exposure to flavonol rich red wine. Life Sci. 71:2881-95.

29. Bertelli AAE et al. (2005) Effect of ethanol and red wine on ochratoxin A-induced experimental acute nephrotoxicity. J. Agric. Food. Chem. 53:6924-9.

30. Laemmli UK. (1970) Cleavage of structural proteins during the assembly of the head of bacteriophage T4. Nature 227:680-5.

31. Simon P, Godin M, Fillastre JP. (1996) Ochratoxin a: a new environmental facto which is toxic for the kidney? Nephrol. Dial. Transplant. 11:2389-91.

32. Kane A, Creppy EE, Roschenthaler R, Dirheimer G. (1986) Changes in urinary and renal tubular enzymes caused by subchronic administration of ochratoxin A in rats. Toxicology 42:233-43.

33. Gagliano N et al. (2000) Age-dependent expression of fibrosis-related genes and collagen deposition in rat kidney cortex. J. Gerontol. 55:B365-72.

34. Steinmann-Niggli K, Ziswiler R, Kung M, Marti HP. (1998) Inhibition of metalloproteinases attenuates anti-Thyl.1 nephritis. J. Am. Soc. Nephrol. 9:397-407.

35. Sato Y, Fujimoto S, Hamai K, Eto T. (1998) Serial alterations of glomerular matrixdegrading metalloproteinase activity in anti-thymocyte-induced glomerulonephritis in rats. Nephron 78:195-200.

36. Sakai T, Gross J. (1967) Some properties of the products of reaction of tadpole collagenase with collagen. Biochemistry 6:518-28.

37. Walker LC, Overstreet MA, Yeowell HN. (2005) Tissue-specific expression and regulation of the alternatively-spliced forms of lysyl hydroxylase 2 (LH2) in human kidney cells and skin fibroblasts. Matrix Biol. 23:515-23.

38. van der Slot AJ et al. (2004) Increased formation of pyridoline cross-links due to highetr telopeptide lysyl hydroxylase levels is a general fibrotic phenomenon. Matrix Biol. 23:251-7.

39. Yu L, Border WA, Huang Y, Noble NA. (2003) TGF- $\beta$ isoforms in renal fibrogenesis. Kidney Int. 64:844-56.

40. German JB, Walzem RL. (2000) The health benefits of wine. Annu. Rev. Nutr. 20 561-93.

41. Abdel-Wahhab MA, Abdel-Galil MM, El-Lithey M. (2005) Melatonin counteracts oxidative stress in rats fed an ochratoxin A contaminated diet. J. Pineal Res. 38:130-5.

42. Ozcelik N, Soyoz M, Kilink I. (2004) Effects of ochratoxin A on oxidative damage in rat kidney: protective role of melatonin. J. Appl. Toxicol. 24:21 1-5.

43. Sauvant C, Holzinger H, Mildenberger S, Gekle M. (2005) Exposure to nephrotoxic ochratoxin A enhances collagen secretion in human renal proximal tubu lar cells. Mol. Nutr. Food. Res. 49:31-7.

44. Zeisberg M, Kalluri R. (2004) The role of epithelial-to-mesenchymal transition in renal fibrosis. J. Mol. Med. 82:175-81.

45. Masszi A et al. (2004) Integrity of cell-cell contacts is a critical regulator of TGF- $\beta 1$-induced epithelial-to-myofibroblast transition. Am. J. Pathol. 165:1955-67.

46. Nouwen EJ, Dauwe S, van der Biest I, De Broe ME. (1993) Stage- and segmentspecific expression of cell-adhesion molecules N-CAM, A-CAM, and L-CAM in the kidney. Kidney Int. 44:147-58.

47. Nadasdy T, Laszik Z, Blick KE, Johnson DL, Silva FG. (1994) Tubular atrophy in the end-stage kidney: a lectin and immunohistochemical study. Hum. Pathol. 25:22-8.

48. Horster MF, Braun GS, Huber SM. (1999) Embryonic renal epithelia: induction, nephrogenesis, and cell differentiation. Physiol. Rev. 79:1157-91.

49. Wheelock MJ, Johnson KR. (2003) Cadherins as modulators of cellular phenotype. Annu. Rev. Cell. Dev. Biol. 19:207-35.

50. Collins JE et al. (1995) Regulation of desmocollin transcription in mouse preim plantation embryos. Development 121:743-53.

51. Matsumoto K, Nakamura T. (2001) Hepatocyte growth factor: renotropic role and potential therapeutics for renal diseases. Kidney Int. 59:2023-38. 